\title{
Introduction to Structural and Functional Studies on Nerve Growth Factor
}

Nerve growth factor (NGF) was discovered more than five decades ago by Rita Levi-Montalcini while working at Washington University in Viktor Hamburger's laboratory. It is a pleasure for us to contribute to the celebration of Rita LeviMontalcini's 90 birthday with the publication of this issue of Microscopy Research and Technique. Her contribution to NGF research is outlined in a short biographical introduction written by her colleague Luigi Aloe (1999a) and her ideas on NGF actions at this moment are presented by her in her Preface. The Instituto of Neurobiología Santiago Ramón y Cajal of Madrid (Spain) has collaborated in this homage by contributing the photograph of Rita Levi-Montalcini receiving the Cajal Medal.

NGF was initially characterized by its trophic role, including the prevention of cell death by apoptosis in specific populations of neurons in the peripheral nervous system. NGF is the first identified member of a family of factors, called neurotrophins, that promote neuronal survival in both the central and the peripheral nervous systems. This neurotrophin family also includes the brain-derived neurotrophic factor (BDNF), neurotrophin (NT) 3 and NT-4/5. All members of the neurotrophin family are known to bind two receptors, p75NFR, that binds neurotrophins with low affinity; and one trk receptor. Within the family of tyrosine kinase receptor trks, three members have been described. Trk $A$ that binds NGF, trkB that binds BDNF and NT-4/5 and trkC that binds NT-3. The role of

neurotrophins in neuronal survival is mainly mediated by the activation of the trk receptors. The signaling pathways elicited by trk receptors are reviewed by Klesse and Parada (1999). The role of neurotrophins and their receptors during neural development is presented in three chapters. Fariñas (1999) as well as Mendell et al., (1999), review rodent models; Frade et al., (1999) focus on a avian model of neural development. Because neurotrophins do not account for the survival of all neuronal types during development, we have also included in this issue another family of proteins. Saarma and Sariola (1999) discuss the in vivo effects of the GDNF family of growth factors. As we mentioned above, neurotrophins also bind the low affinity receptor p75. In spite of a wealth of literature, the biological function of this receptor has been elusive. In this issue, Casaccia-Bonnefil et al., (1999) discuss the role of p75 as a modulator of survival and death decisions.

In addition to its survival effects, NGF also regulates other aspects of the neuronal function. Mendell et al., (1999) discuss the role of NGF on nociceptors and Behrens et al., (1999) present evidence that neurotrophins may enhance injury under certain conditions. Furthermore, in his chapter, Tonra (1999) delineates the importance of a new mode of neurotrophin action that may be involved in the neuronal response to injury, inflammation and pain. And Griesback et al., (1999) present data about the characteristics of NGF and BDNF secretion that may modulate activity-dependent neuronal plasticity. 
The role of NGF on non-neuronal cells was first described by Dr. Levi-Montalcini in the late seventies. Her observations have been expanded and are leading to the discovery of new biological actions for this family of neurotrophins. For instance, the role of NGF as an important regulator of the immune system is reviewed Aloe et al., (1999b) in this special issue.

ISABEL VARELA-NIETO1 AND INMACULADA SILOS-SANTIAGO2

1 Instituto de Investigaciones Biomédicas Alberto Sols, CSIC-UAM, Madrid 28029, Spain

2Deptartment of Neurobiology, Millennium Pharmaceuticals Inc., Cambridge, MA 02139 